\title{
Application of Novel Techniques to the Three-dimensional Characterization of Microstructural Features in $\alpha+\beta$ Titanium Alloys
}

\author{
J.M. Sosa, ${ }^{*}$ S. Koduri, ${ }^{*}$ V. Dixit, ${ }^{*}$ P.C. Collins, ${ }^{* *}$ S. Niezgoda, ${ }^{* * *}$ S.R. Kalidindi, ${ }^{* * *}$ \\ H.L. Fraser* \\ * Center for the Accelerated Maturation of Materials, The Ohio State University, 2041 \\ College Rd., 477 Watts Hall, Columbus, OH 43210 \\ ** Quad City Manufacturing Lab \& Western Illinois University-Quad Cities, 2408 4th \\ Ave., Rock Island, IL 61201 \\ *** Department of Materials Science and Engineering, Drexel University, 3141 Chestnut \\ Street, Philadelphia, PA 19104
}

Advanced three-dimensional data collection techniques such as Robo.Met-3D ${ }^{\mathrm{TM}}$ has led to rapid acquisition of robust datasets on optical length scales. Implementation of such datasets may improve the accuracy of neural networks and phase-field models. However, the accurate statistical representation of three-dimensional microstructural features is challenging and thus requires further improvement to analytical methods. This work addresses the serial two-dimensional collection, three-dimensional processing, and analysis of datasets containing microstructural features such as equiaxed- $\alpha$ and colony- $\alpha$ in $\alpha+\beta$ titanium alloys.

In regards to equiaxed- $\alpha$, new algorithms, including a novel 3-D adaptive threshold, have been developed for the three-dimensional identification and isolation of equiaxed- $\alpha$ particles from the $\alpha+\beta$-processed microstructure. Furthermore, innovative, automated separation subroutines have been constructed and applied to the identified equiaxed- $\alpha$ particles. Such subroutines have resulted in three-dimensional separation of particle clusters into several discrete features that were falsely joined by segmentation artifacts from less robust algorithms (see FIG. 1). Made possible by accurate particle identification and separation, as well as the development of a MATLAB-based toolkit, 3$\mathrm{D}$ quantification was performed on a global and feature-by-feature basis. The extracted 3-D measurements were compared to their 2-D counterparts, leading to an exploration of the representative volume element (RVE) and its dependence on the measurement of interest.

With regard to colony- $\alpha$, a large 3-D dataset of Ti-5111 has been collected using Robo.Met-3 $\mathrm{D}^{\mathrm{TM}}$ and subsequently aligned with high fidelity using the afore-mentioned MATLAB-based toolkit. Through collaboration with Dr. Surya Kalidindi and his group at Drexel University, the dataset was automatically segmented into the various colony orientations using two-point correlation. Several colony orientations were then threedimensionally reconstructed, allowing for the unique 3-D visualization of their interpenetrating morphology (see FIG. 2). Crystallographic information has been incorporated using electron backscatter diffraction (EBSD) and was subsequently integrated into 3-D quantification of the reconstructed colonies. 

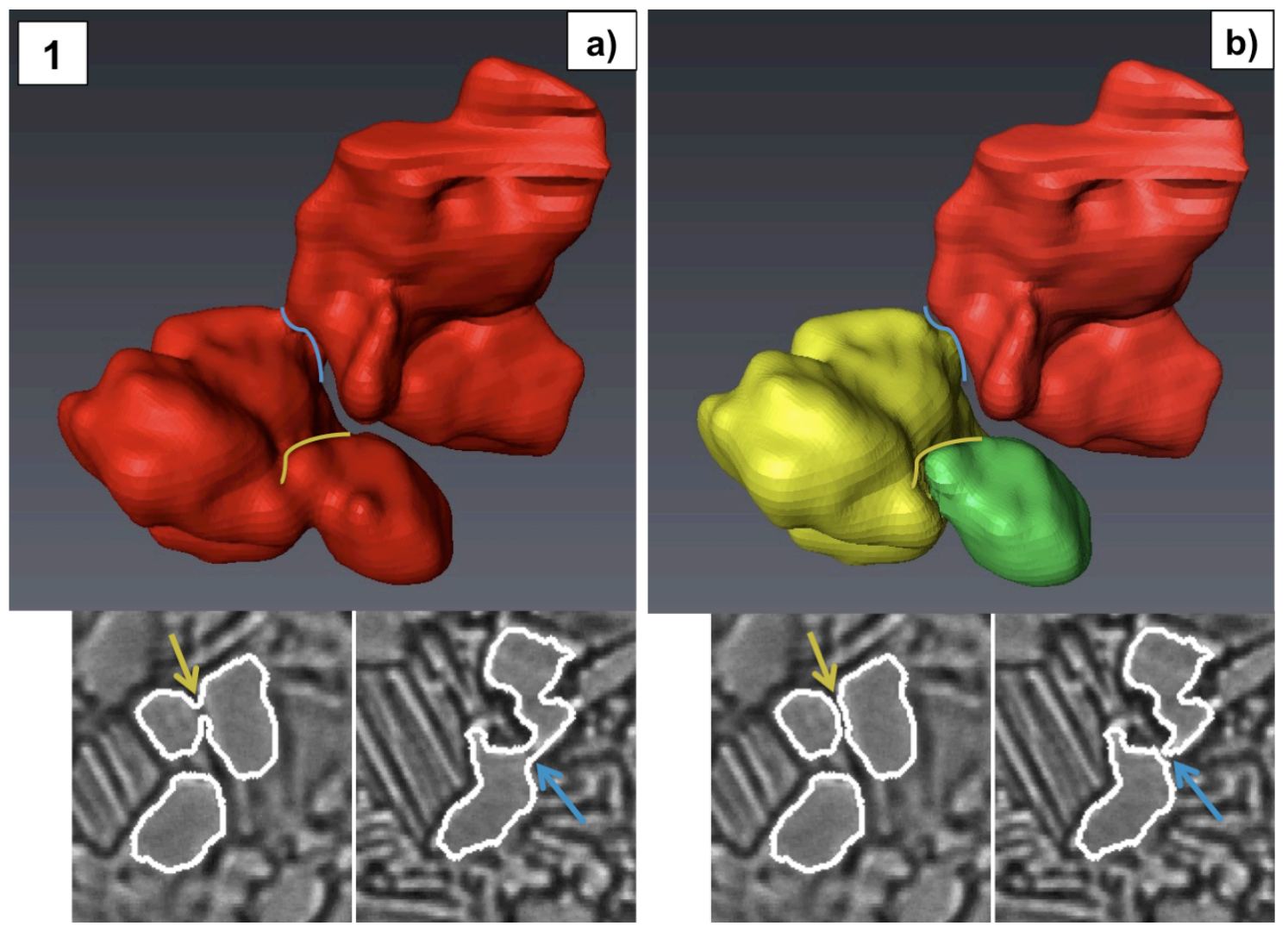

FIG. 1. An example of automated 3-D equiaxed- $\alpha$ particle separation showing an a) unseparated cluster of three particles and b) the separated particles from a novel 3-D separation algorithm.

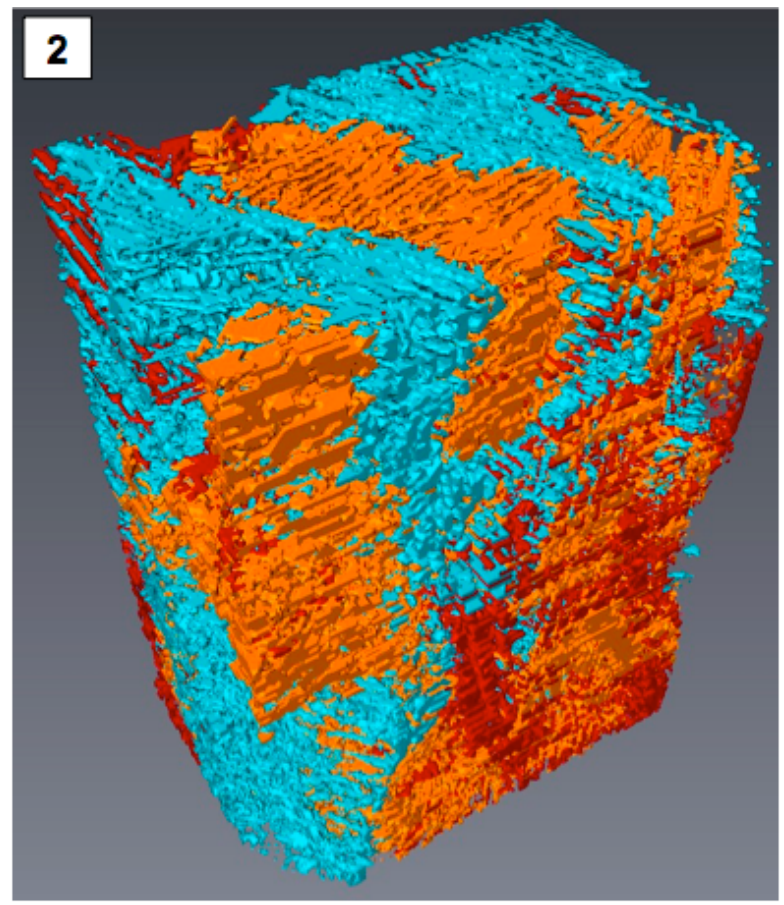

FIG. 2. A 3-D reconstruction of three colony orientations revealing their interpenetrating morphology. Each color indicates a discrete orientation. 